\title{
Analysis of the neuromuscular responses during two different high- intensity interval training sessions in athletes of cyclic and acyclic sports
}

\section{Análisis de las respuestas neuromusculares durante dos diferentes sesiones de entrenamiento intervalado de alta intensidad en atletas de deportes cíclicos y acíclicos}

\author{
1 Universidad de León, España \\ ${ }^{2}$ Universidad Católica de Valencia "San Vicente Mártir", España \\ * Correspondence: Email: diegowarr@hotmail.com. Teléfono: ++34 655722537. \\ Dirección postal: Pda. La Gronsa Pol 10, nº 540 - (46591) Albalat dels Tarongers, \\ Valencia, España.
}

Diego Warr ${ }^{1 *}$, José Vicente Sánchez-Alarcos ${ }^{2}$, Carlos Pablos $^{2}$, Juan Carlos Redondo ${ }^{1}$
Short Title:

Neuromuscular responses during high-intensity interval training sessions

HOW TO CITE THIS ARTICLE:

Warr, D., Sánchez-Alarcos, J.V., Pablos, C., \& Redondo, J.C. (2022). Analysis of the neuromuscular responses during two different high-intensity interval training sessions in athletes of cyclic and acyclic sports. Cultura, Ciencia y Deporte, 17(51), 131-143. http://dx.doi.org/10.12800/ccd.v17i51.1711

\section{Abstract}

The purpose of the present study was to investigate the acute effects of two high-intensity interval training (HIIT) sessions on countermovement jump performance. Twentytwo male regional-level athletes (cyclic, $\mathrm{n}=11$; acyclic, $\mathrm{n}=11$ ) completed two HIIT sessions consisted of multiple running bouts on a treadmill. A different work-interval duration was applied in each session. To determine the effect of fatigue on jumping performance, countermovement jump tests were executed pre-effort and at the end of all series. The results showed a decrease in jump performance at the end of the third series respect to the pre-effort condition $(p<.001)$. In addition., the acyclic athletes jumped higher than the cyclic ones $(p<.001)$, revealing a different jumping strategy based on a higher velocity component, and a more efficient elastic energy utilization $(p<.01)$. The neuromuscular mechanisms affected by fatigue, were also different between groups. In conclusion, during HIIT sessions the neuromuscular status is principally conditioned by the total volume rather than by the work-interval duration, affecting specific performance variables depending on the sport discipline. The present findings may be useful when conducting HIIT concurrently with other training contents in athletes of both cyclic and acyclic sports.

Keywords: Countermovement jump, explosive strength, intermittent exercise, vertical ground reaction force..

\section{Resumen}

El propósito del presente estudio ha sido investigar los efectos agudos de dos sesiones de entrenamiento intervalado de alta intensidad (HIIT) sobre el rendimiento en el salto contramovimiento. Veintidós atletas hombres, de nivel regional (cíclicos, $\mathrm{n}=11$; acíclicos, $\mathrm{n}=11$ ) completaron dos sesiones de HIIT, que consistían en series repetidas de carrera sobre un tapiz rodante. Se aplicaron intervalos de trabajo de diferente duración en cada sesión. Para determinar el efecto de la fatiga sobre el rendimiento en el salto, se ejecutaron pruebas de salto contramovimiento antes del esfuerzo y al final de todas las series. Los resultados mostraron una disminución en el rendimiento del salto a final de las tercera serie respecto a la condición pre-esfuerzo $(p<.001)$. Asimismo, los atletas acíclicos saltaron más alto que los cíclicos, $(p<.001)$, revelando una estrategia de salto diferente, basada en un mayor componente de velocidad y una utilización más eficiente de la energía elástica $(p<.01)$. Los mecanismos neuromusculares afectados por la fatiga también fueron diferentes entre los grupos. En conclusión, durante sesiones de HIIT, el estatus neuromuscular está principalmente condicionado por el volumen total, más que por la duración del intervalo de trabajo, afectando variables específicas dependiendo de la disciplina deportiva. Los presentes hallazgos pueden ser de utilidad cuando se prescribe HIIT de manera concurrente a otros contenidos de entrenamiento tanto en atletas de deportes cíclicos como acíclicos.

Palabras clave: Salto contramovimiento, fuerza explosiva, ejercicio intermitente, fuerza de reacción vertical.. 


\section{Introduction}

Physical conditioning programs should consider the various requirements of a given sport in order to maximize the efficacy of the training process. An appropriate selection of the methodology may help to concurrently enhance a specific performance parameter (i.e. endurance) without diminishing another (i.e. power) (Wilson et al., 2012). In this regard, high-intensity interval training (HIIT) has been demonstrated to be effective in activating central and peripheral components of $\mathrm{VO}_{2}$ (Zafeiridis et al., 2015) as well as minimizing metabolic and cardiorespiratory responses at the same intensity compared with continuous prescriptions (Billat et al., 2000; Tschakert et al., 2015). This method consists of executing bouts of exercise at an elevated intensity intercalated with recovery periods of rest or low-intensity activity (Buchheit \& Laursen, 2013; Tschakert \& Hofmann, 2013). In addition, this kind of protocols can be used to elicit precise metabolic responses by modulating load components such as the intensity (Wakefield \& Glaister, 2009), workinterval duration (Price \& Moss, 2007; Warr-di Piero et al., 2018) and/or work-to-rest ratio (Rozenek et al., 2007).

Similarly, it is important to analyze neuromuscular responses associated with fatigue. For this purpose, jump tests are practical and non-invasive assessments used for monitoring athletic performance (Cormie et al., 2010; Harry et al., 2018), and comparing the effects of training programmes (Griffiths et al., 2019; Vasquez-Bonilla et al., 2021). Specifically, countermovement jump (CMJ) has proven to be sensitive in detecting fatigue or supercompensation effects (BenítezJiménez et al., 2020; Claudino et al., 2017) and it also presents a high level of reliability of measures during assessments performed on a force platform (Hori et al., 2009; Warr et al., 2020).

Previous studies have analyzed acute neuromuscular fatigue focusing on several parameters of jump performance (Bedo et al., 2020; Benjaminse et al., 2008; Cormack et al., 2008; Gathercole et al., 2015; Watkins et al., 2017). One study found that a stiff landing strategy was used following an incremental protocol until exhaustion (Benjaminse et al., 2008), marked by less knee valgus and knee flexion at initial contact of a single-leg-stop jump task. An altered movement strategy in CMJ was also reported after two consecutive intermittent protocols until exhaustion, suggesting that a full CMJ-variables battery is a more prudent approach to detect neuromuscular fatigue (Gathercole et al., 2015). Cormack et al. (2008) examined the acute responses in single and repeated CMJ after an elite Australian Rules Football match and found a decrease in the reactive strength index in the post-match condition.

These findings indicate that neuromuscular status can be affected by efforts performed until exhaustion. However, the effects that high-intensity interval training protocols could have on neuromuscular status remains unclear. Furthermore, to the best of our knowledge, no studies have analyzed the neuromuscular responses between interval protocols with different configurations (i.e. equal total load but different work-interval durations). Therefore, the aim of the present study was to analyze the acute effects of a short and a long HIIT session on CMJ performance in athletes of cyclic and acyclic sports. It was hypothesized that i) an increase of the relative volume would result in a diminution of the jump performance, ii) a HIIT session with long work-interval durations would evoke neuromuscular fatigue earlier than a session with short intervals, and iii) the jumping profile, and the neuromuscular responses to HIIT displayed by cyclic athletes would be different than those displayed by acyclic athletes.

\section{Materials and Methods}

\section{Experimental Overview}

Each participant completed a total of four testing sessions separated at least by 72 hours. All the tests were executed in the sports science laboratory, with environmental conditions of $21.8 \pm 0.6^{\circ} \mathrm{C}, 39 \pm 10 \%$ humidity and $1012 \pm 10 \mathrm{hPa}$. The participants were required to attend the laboratory in their usual training clothes and wearing running shoes. A complete recovery condition was requested before starting all the sessions, which consisted of no previous physical activity during the 48 hours before testing, a minimum of 7 hours of sleep the night before, a carbohydrate-rich diet, avoidance of any food, tobacco, caffeine or supplement intake for at least 3 hours prior to testing, and drinking water regularly during the testing day. In the first session, a maximal incremental running test was completed to determine maximal oxygen uptake $\left(\mathrm{VO}_{2} \mathrm{max}\right)$ and maximal aerobic speed (MAS). In the second session, a time to exhaustion test was executed. Based on these data, two HIIT protocols were designed and performed in sessions 3 and 4 . Both experimental protocols were equal in terms of relative intensity, volume and density but differed in the work-interval duration. In consequence, short and long HIIT protocols were defined. To assess the effect of the type of protocol on neuromuscular fatigue, CMJ tests were conducted in each experimental session.

\section{Participants}

Twenty-two male regional-level athletes participated in the study. Based on the previous study by Warr-diPiero et al. (2018), an a priori power analysis (G*Power3) with $a<0.05$ and $1-\beta=80$ indicated that a sample size of at least 18 was required to explore neuromuscular responses associated with HIIT in athletes of cyclic and acyclic sports. The participants were all experienced athletes with a minimum of 3 years of regular participation in competitions, and a training frequency of $4.5 \pm 1.1$ sessions per week. According to the characteristics of their sport, participants were classified into the cyclic group (middle- and long-endurance runners, $n=11,20.3 \pm 3.2$ years, $176.2 \pm 7.0 \mathrm{~cm}, 64.7 \pm 6.7 \mathrm{~kg}, 8.7 \pm 3.1 \%$ fat mass) or the acyclic group (team sports players: handball, $n=4$; football, $\mathrm{n}=4$; field hockey, $\mathrm{n}=3,20.8 \pm 3.2$ years, $181.2 \pm 8.3 \mathrm{~cm}$, $72.0 \pm 7.0 \mathrm{~kg}, 10.1 \pm 3.0 \%$ fat mass). This classification applied only for data analysis, so all the participants completed the same intervention protocol. A complete written and verbal description of the research protocol was given to the volunteers. Afterwards, the participants signed an informed consent form prior to any further intervention. The study was approved by the University's Ethics Committee (ETICAULE-022-2021).

\section{Procedures}

All running tests were conducted on a motorized treadmill (H/P Cosmos Quasar, Cosmos Sports \& Medical, Nussdorf-Traunstein, Germany) graded at a $1 \%$ inclination. The participants were equipped with a portable gas analyzer (Oxycon Mobile, CareFusion, Hoechberg, Germany) to measure gas exchange breath-by-breath during the 
incremental test. Heart rate (HR) was recorded throughout all sessions at a sampling frequency of $1 \mathrm{~Hz}$ using a Polar RS800CX heart rate monitor (Polar Electro Oy, Kempele, Finland). Capillary blood samples were taken from the participants' earlobes before and one minute after completion of the incremental test to determine blood lactate concentrations using a Pro-Lactate 2 analyzer (Arkray Inc. Kyoto, Japan). All CMJ tests were performed on a force plate (Kistler 9281EA, Kistler Instrument AG, Winterthur, Switzerland) connected to computer software (Bioware 5.3). The vertical ground reaction force (VGRF) was recorded at a sampling frequency of $1000 \mathrm{~Hz}$ over a period of $10 \mathrm{~s}$ for each trial. Then, the data were downloaded and saved for subsequent analysis. The participants had to complete a total of 3 valid trials of CMJs separated by 30 s of recovery for every jump testing condition. The average of each set of three trials was considered as the value for a given condition (pre-effort and inter-series) in order to increase sensitivity in detecting of fatigue effects (Claudino et al., 2017). Participants started all sessions by performing a standardized warm-up (Warr et al., 2020). CMJ familiarization sets were implemented in sessions 1 and 2, in order to assure correct execution, and avoid learning effects during the experimental HIIT sessions.

\section{Performance Assessments}

The Maximal Incremental Running Test started at an initial speed of $10.0 \mathrm{~km} \cdot \mathrm{h}^{-1}$ and increased by $0.5 \mathrm{~km} \cdot \mathrm{h}^{-1}$ each minute until exhaustion. Oxygen uptake was considered maximal if at least 3 of the recommended criteria were met (Wakefield \& Glaister, 2009; Zafeiridis et al., 2015). The speed of the stage in which $\mathrm{VO}_{2 \max }$ have been achieved was recorded as MAS The Time to Exhaustion Test ( $T_{\text {LIM }}$ ) consisted of running at MAS until volitional exhaustion (Dupont et al., 2002). TLIM waS registered in seconds.

\section{HIIT protocols}

The results obtained in the performance assessments allowed us to design the two experimental HIIT protocols, which were performed in a random and counterbalanced manner in sessions 3 and 4 to avoid order effects. The intensity of both protocols was equal to MAS and the work-to-rest ratio was 1:1. The total volume of each protocol was related to $T_{\text {LIM }}$, so that each HIIT protocol constituted a total volume equal to three times the volume reached in the $T_{\text {LIM }}$ test. The workinterval duration determined the type of protocol and was also related to the $\mathrm{T}_{\text {LIM }}$ test. In this way, the work-interval duration of the short HIIT protocol was $5 \%$ of $T_{\text {LIM }}\left(T_{\text {LIM-5 }}\right)$ and the work-interval duration of the long HIIT protocol was 33\% of $T_{\text {LIM }}\left(T_{L I M}-33\right)$. Therefore, a total of 60 and 9 repetitions had to be completed in the short and long protocols, respectively. In addition, to analyze whether the increase of volume could trigger an inflection point due to fatigue accumulation, the protocols were conducted in 3 equal series $(3 \times 20$ repetitions for the short protocol and $3 \times 3$ repetitions for the long one) between which CMJ performance was assessed, immediately after de cessation of the efforts. The duration of the interseries measurements was fixed at 5 minutes for all subjects. A flow chart of the design is presented in Figure 1. 


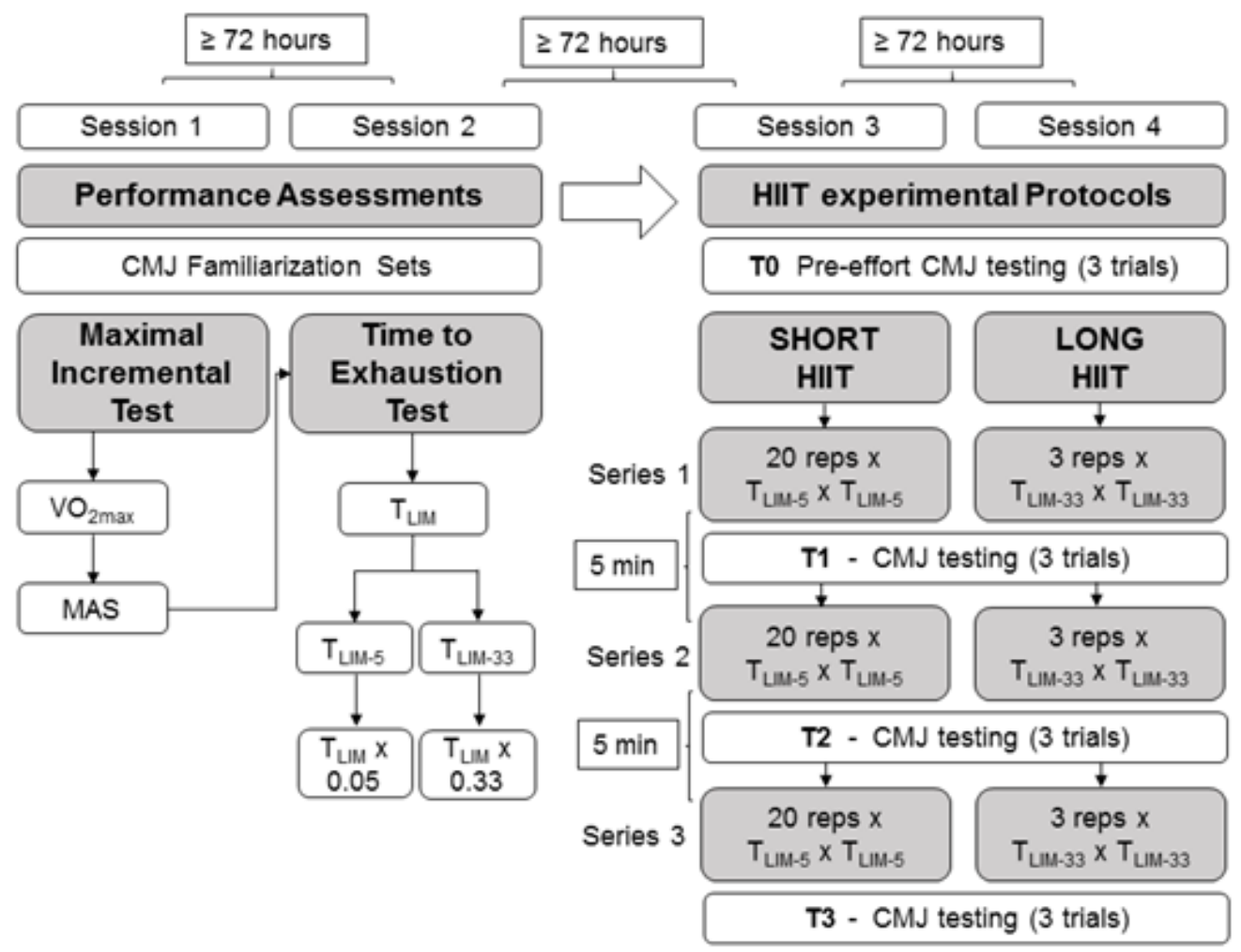

Figure 1. Schematic overview of the four sessions

HIIT = high-intensity interval training, MAS = maximal aerobic speed, $\mathrm{T}_{\mathrm{LIM}}=$ time to exhaustion, reps $=$ repetitions, CMJ = countermovement jump; $\mathrm{T0}=$ pre-effort condition; $\mathrm{T} 1$ = post-series 1 condition; $\mathrm{T} 2$ = post-series 2 condition; $\mathrm{T} 3$ = post-series 3 condition.

\section{Data Processing}

The VGRF data from all the trials were included in the analysis. Basic calculations were conducted to obtain instantaneous values of velocity, displacement and power for each trial (Harry et al., 2018; Kirby et al., 2011; Warr et al., 2020).

Key points and determination of phases

Several points of interest were marked for the further determination of the different phases and sub-phases of the jump (Figure 2). The body weight (BW) was determined as the mean force of a two-second standing still stance before the jump initiation. The force value at jump initiation ( $\left.F_{\text {START }}\right)$ was determined as BW minus 5 times the standard deviation of the two-second stance. The minimum force $\left(F_{M I N}\right)$ was the lowest force value registered before reaching $B W$. The force value at minimum velocity was $\mathrm{F}_{\mathrm{BRAKE}}$. The countermovement amplitude ( $D_{\text {MAX }}$ ) determined the initiation of propulsive subphase, and the force value at this point was FPROP. 


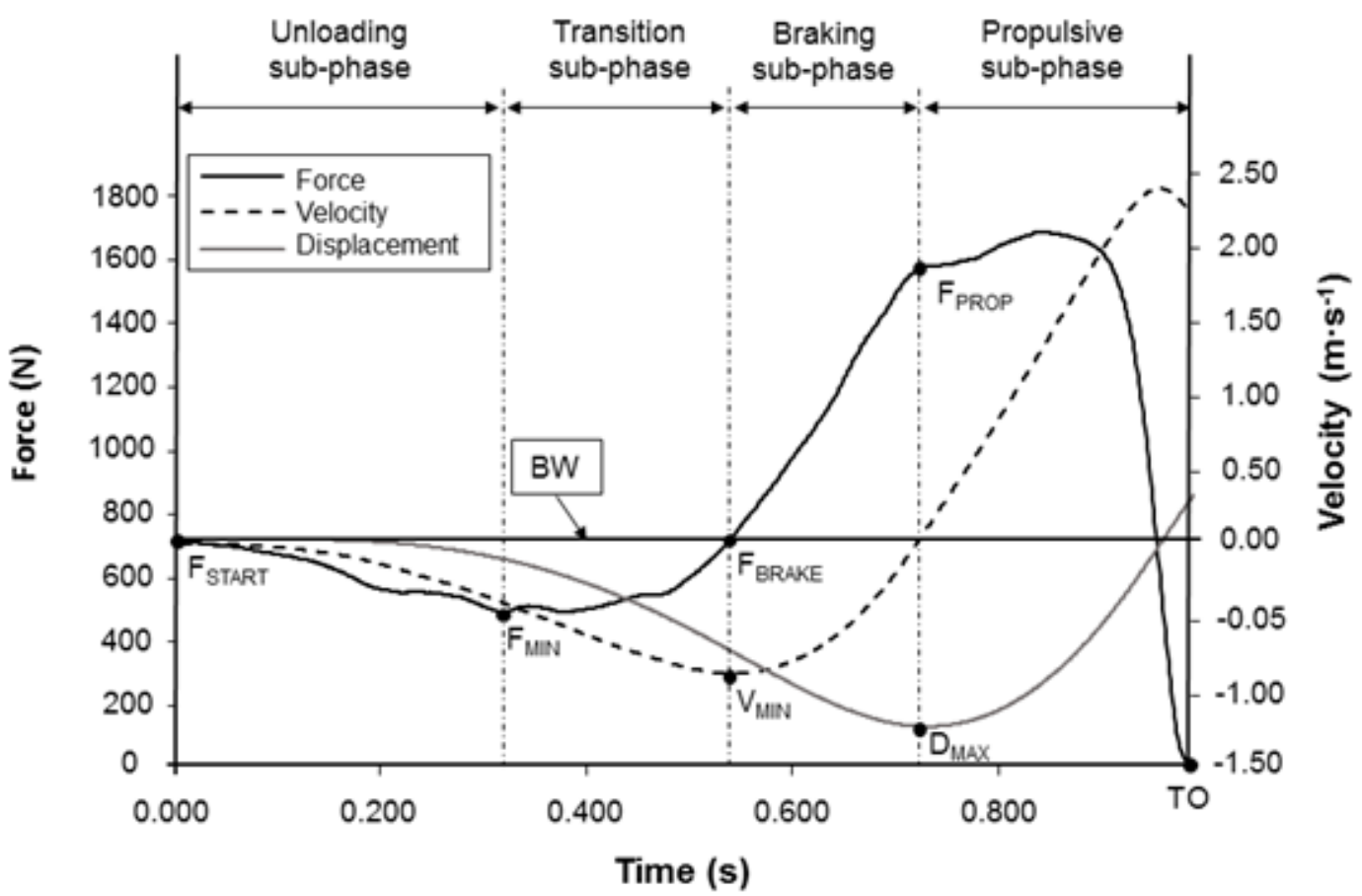

Figure 2. Key points and CMJ sub-phases of an example trial of one subject

$\mathrm{BW}=$ body weight, $\mathrm{F}_{\mathrm{START}}=$ force at jump initiation, $\mathrm{F}_{\mathrm{MIN}}=$ minimum force, $\mathrm{FB}_{\mathrm{RAKE}}=$ force at minimum velocity, $\mathrm{FPROP}_{\mathrm{P}}=$ force at the beginning of the propulsive sub-phase, $\mathrm{V}_{\mathrm{MIN}}=$ minimum velocity, $\mathrm{D}_{\mathrm{MAX}}=$ countermovement amplitude, $\mathrm{TO}=$ take-off.

Note: The displacement scale has been omitted.

Calculation of Performance Variables

Time-, force-, power- and velocity-related variables, as well as net impulse and rates of force development, were calculated for the different sub-phases of the jump. A description of the variables is summarized in Table 1. 
Table 1. CMJ performance variables

\begin{tabular}{|c|c|}
\hline Variables (Abbreviation) & Description \\
\hline Flight time (FT) & $\begin{array}{l}\text { Time from take-off to landing. Both points were determined } \\
\text { by the same force value threshold (mean force of a } 30 \text {-ms } \\
\text { period during the flight minus } 5 \text { times the SD of the same } \\
\text { period). }\end{array}$ \\
\hline Jump height (JH) & $1 / 2 \mathrm{~g}(\mathrm{FT} / 2)^{2}$, where $\mathrm{g}=9.81 \mathrm{~m} / \mathrm{s}^{2}$ (Moir, 2008). \\
\hline Contact time (TCONTACT) & Time from jump initiation to take-off. \\
\hline Unloading time (TUNLOAD) & Duration of the unloading sub-phase. \\
\hline Transition time (TTRANSITION) & Duration of the transition sub-phase. \\
\hline Braking time ( $\left.\mathrm{T}_{\mathrm{BRAKE}}\right)$ & Duration of the braking sub-phase. \\
\hline Propulsive time (TPROP) & Duration of the propulsive sub-phase. \\
\hline Peak force (PF) & Highest force value achieved. \\
\hline Mean unloading force (MFUNLOAD) & Average force during the unloading sub-phase. \\
\hline Mean transition force (MF TRANSImoN) & Average force during the transition sub-phase. \\
\hline Mean braking force (MFBRAKE) & Average force during the braking sub-phase. \\
\hline Mean propulsive force (MFPROP) & Average force during the propulsive sub-phase. \\
\hline Minimum power $\left(\mathrm{P}_{\mathrm{MN}}\right)$ & Lowest power value generated during the jump. \\
\hline Peak power $(\mathrm{PP})$ & Highest power value achieved during the jump. \\
\hline Mean transition power (MPTRANSITION) & Average power of the transition sub-phase. \\
\hline Mean braking power (MPBRAKE) & Average power of the braking sub-phase. \\
\hline Mean propulsive power (MPpRop) & Average power of the propulsive sub-phase. \\
\hline Minimum velocity ( $\mathrm{VMN})$ & Lowest jump velocity (peak negative velocity). \\
\hline Peak velocity (PV) & Highest velocity value achieved. \\
\hline Velocity at peak power (Vpp) & Velocity at the point where PP was registered. \\
\hline Mean transition velocity (VTRANSITION) & Average velocity of the transition sub-phase. \\
\hline Mean braking velocity (VBRAKE) & Average velocity of the braking sub-phase. \\
\hline Braking net impulse (NIBRAKE) & Net impulse calculated for the braking sub-phase. \\
\hline Propulsive net impulse (NIPROP) & Net impulse calculated for the propulsive sub-phase. \\
\hline Unloading RFD (RFDUNLOAD) & $\left(F_{M M N}-F_{S T A R T}\right) /$ TUNLOAD \\
\hline Transition RFD (RFDTRANSITION) & $\left(F_{\text {BRAKE }}-F_{M I N}\right) / T_{\text {TRANSITION }}$ \\
\hline Braking RFD (RFDBRAKE) & $\left(F_{P R O P}-F_{B R A K E}\right) / T_{\text {BRAKE. }}$ \\
\hline
\end{tabular}

$\mathrm{SD}=$ standard deviation, RFD = rate of force development, $\mathrm{F}_{\mathrm{MIN}}=$ minimum force, FSTART = force at jump initiation, FBRAKE = force at the beginning of the braking subphase, $F_{P R O P}=$ force at the beginning of the propulsive sub-phase.

$\mathrm{SD}=$ standard deviation, RFD = rate of force development, $\mathrm{F}_{\mathrm{MIN}}=$ minimum force, $\mathrm{F}_{\mathrm{START}}=$ force at jump initiation, $F_{B R A K E}=$ force at the beginning of the braking sub-phase, $F_{P R O P}=$ force at the beginning of the propulsive sub-phase.

\section{Statistical Analysis}

Statistical Package for Social Sciences (version 24.0, SPSS, Inc., Chicago, IL, USA) was used to analyze all the data. The Kolmogorov-Smirnoff test was used to confirm the normal distribution of the data. A single-factor ANOVA test was used to detect differences in the descriptive data between the cyclic and acyclic groups. A separate general linear repeated measures ANOVA model was used to determine effects on the performance variables, with "protocol" (short, long) and "time points" (T0, T1, T2, T3) as within-subject factors, and "discipline" (cyclic, acyclic) as the inter-subject factor. Assumptions of the test statistics were verified with the Mauchly test of sphericity. If significant effects were observed, a Bonferroni post-hoc test was undertaken. Partial

$$
\text { Cultura, Ciencia y Deporte | AÑ̃o } 2022 \text { | volumen } 17 \text { | NUM. } 51 \text { | España | PÁG. } 131 \text { A } 143 \text { | ISSN: 1696-5043 }
$$


eta-squared $\left(\eta_{p}{ }^{2}\right)$ and observed power were used to interpret the magnitude of the main effects. The relative reliability of all the variables was assessed with the intraclass correlation coefficient (ICC) from a two-way mixed-effects model with a mean of . measurements and absolute agreement definition. Statistical significance was set at an a level of 0.05 .

\section{Results}

All the variables exhibited a normal distribution and displayed high reliability values, expressed in ICCs ranging from 0.85 to 0.99 .

The one-factor ANOVA indicated that there were significant differences in the anthropometric data and performance assessments between sport disciplines. The acyclic group showed a significantly greater mass than the cyclic one $(F$ $=6.34, p=.020$ ); consequently, further force, power and impulse comparisons between groups were conducted in relative terms. In the performance assessments, no significant differences between groups were observed in either absolute $\mathrm{VO}_{2 \max }(\mathrm{F}=0.06, \mathrm{p}=.941)$ or relative $\mathrm{VO}_{2 \max }(\mathrm{F}=3.21$, $p=.090)$. However, an effect of the sport discipline was detected on MAS $(F=39.17, p<.001)$, with the cyclic athletes reaching a significantly higher speed $\left(17.7 \pm 1.3 \mathrm{~km} \cdot \mathrm{h}^{-1}\right)$ than the acyclic ones $\left(14.3 \pm 1.2 \mathrm{~km} \cdot \mathrm{h}^{-1}\right)$. In addition, a nonsignificant difference between disciplines $(F=2.50, p=.130)$ was observed in the time-to-exhaustion test (cyclic group: 408 \pm 91 s; acyclic group: $355 \pm 62 \mathrm{~s}$ ).

A total of $528 \mathrm{CMJ}$ trials were included in the analysis. There were no significant effects of the type of protocol on any of the CMJ performance variables.

\section{CMJ gross performance outcomes}

An effect of the sport discipline was observed on FT and JH ( $F$ $=5.76, p=.026, \eta_{\mathrm{p}}{ }^{2}=0.22$, power $=0.63$ and $F=6.64, p=.018$, $\eta_{\mathrm{p}}{ }^{2}=0.25$, power $=0.69$, respectively). The acyclic athletes developed a significantly longer flight time and, therefore, a higher jump than the cyclic ones. A main effect of time was also observed on these measures (Table 2). Post-hoc testing revealed that in the short protocol the acyclic group showed a significantly lower $\mathrm{JH}(p=.041)$ and a significantly shorter flight time $(p=.047)$ in T3 than in the pre-effort condition.

Table 2. Gross performance outcomes Values are expressed as mean \pm SD

\begin{tabular}{|c|c|c|c|c|c|c|c|}
\hline \multirow{2}{*}{ Protocol } & \multirow{2}{*}{ Time } & \multicolumn{2}{|c|}{ FT (ms) } & \multicolumn{2}{|c|}{$\mathrm{JH}(\mathrm{cm})$} & \multicolumn{2}{|c|}{ TCONTACT (ms) } \\
\hline & & Cyclic & Acyclic & Cyclic & Acyclic & Cyclic & Acyclic \\
\hline \multirow{4}{*}{ Short } & T0 & $494 \pm 41$ & $534 \pm 29$ & $30 \pm 5$ & $35 \pm 4$ & $944 \pm 204$ & $896 \pm 132$ \\
\hline & $\mathrm{T} 1$ & $488 \pm 38$ & $524 \pm 29$ & $29 \pm 5$ & $34 \pm 4$ & $909 \pm 162$ & $871=121$ \\
\hline & $\mathrm{T} 2$ & $487 \pm 43$ & $524 \pm 33$ & $29 \pm 5$ & $34 \pm 4$ & $939 \pm 133$ & $859 \pm 107$ \\
\hline & T3 & $488 \pm 42$ & $\mathbf{5 2 2} \pm \mathbf{3 0} \S$ & $29 \pm 5$ & $33 \pm 4^{\S}$ & $916 \pm 163$ & $877 \pm 127$ \\
\hline \multirow{4}{*}{ Long } & T0 & $498 \pm 40$ & $530 \pm 46$ & $31 \pm 5$ & $35 \pm 6$ & $968 \pm 187$ & $882 \pm 115$ \\
\hline & $\mathrm{T} 1$ & $493 \pm 30$ & $522 \pm 41$ & $30 \pm 4$ & $34 \pm 7$ & $931 \pm 165$ & $854 \pm 107$ \\
\hline & $\mathrm{T} 2$ & $488 \pm 29$ & $526 \pm 42$ & $29 \pm 3$ & $34 \pm 6$ & $947 \pm 122$ & $871 \pm 92$ \\
\hline & T3 & $483 \pm 29$ & $526 \pm 44$ & $29 \pm 3$ & $34 \pm 7$ & $944 \pm 143$ & $876 \pm 88$ \\
\hline & $p$ & $\eta_{\mathrm{p}}^{2}$ & $p$ & $\eta_{p^{2}}^{2}$ & $P$ & $\eta_{p^{2}}^{2}$ \\
\hline \multicolumn{2}{|l|}{ Protocol } & .940 & 0.00 & .437 & 0.03 & .338 & 0.05 \\
\hline \multicolumn{2}{|l|}{ Time } & $<.001$ & 0.30 & .024 & 0.15 & .060 & 0.14 \\
\hline \multicolumn{2}{|l|}{ Discipline } & .026 & 0.22 & .018 & 0.25 & .297 & 0.06 \\
\hline \multicolumn{2}{|c|}{ Protocol $x$ Time } & .747 & 0.02 & .610 & 0.03 & .534 & 0.04 \\
\hline \multicolumn{2}{|c|}{ Protocol x Discipline } & .772 & 0.00 & .594 & 0.01 & .164 & 0.09 \\
\hline \multicolumn{2}{|c|}{ Time $x$ Discipline } & .631 & 0.03 & .677 & 0.03 & .923 & 0.01 \\
\hline
\end{tabular}

$\S$ Significantly different from T0 $(p<.05)$. Values in bold express significant differences between groups for the given time point $(p<.05)$. FT $=$ flight time; $J \mathrm{H}=$ jump height; $\mathrm{T}_{\text {CONTACT }}$ = contact time; $\mathrm{T} 0$ = pre-effort condition; $\mathrm{T} 1$ = post-series 1 condition; $\mathrm{T} 2$ = post-series 2 condition; $\mathrm{T} 3=$ post-series 3 condition .

Time- and Force -related variables

A significant effect of sport discipline was observed only in the variables associated to the braking sub-phase (Table 3).
Moreover, an interaction between protocol and discipline $(F=$ $5.16, p=.034, \eta_{p}^{2}=0.21$, power $=0.58$ ) was also observed in TBRAKE. 
Table 3. Time- and force -related variables. Values are expressed as mean \pm SD

\begin{tabular}{|c|c|c|c|c|c|}
\hline \multirow{2}{*}{ Protocol } & \multirow{2}{*}{ Time } & \multicolumn{2}{|c|}{$\mathrm{T}_{\mathrm{BRAKE}}(\mathrm{ms})$} & \multicolumn{2}{|c|}{$\mathrm{MF}_{\text {BRAKE }}\left(\mathrm{N} \cdot \mathrm{kg}^{-1}\right)$} \\
\hline & & Cyclic & Acyclic & Cyclic & Acyclic \\
\hline \multirow{4}{*}{ Short } & T0 & $240 \pm 56$ & $208 \pm 51$ & $14.1 \pm 1.8$ & $16.3 \pm 1.4$ \\
\hline & $\mathrm{T} 1$ & $227 \pm 51$ & $201 \pm 28$ & $14.3 \pm 1.5$ & $16.1 \pm 0.9$ \\
\hline & $\mathrm{T} 2$ & $251 \pm 59$ & $205 \pm 34$ & $14.1 \pm 1.6$ & $16.0 \pm 1.1$ \\
\hline & $\mathrm{T} 3$ & $236 \pm 53$ & $208 \pm 30$ & $14.2 \pm 2.0$ & $15.9 \pm 1.0$ \\
\hline \multirow{4}{*}{ Long } & T0 & $265 \pm 111$ & $200 \pm 33$ & $14.0 \pm 2.0$ & $16.5 \pm 2.0$ \\
\hline & $\mathrm{T} 1$ & $255 \pm 75$ & $199 \pm 23$ & $13.8 \pm 1.9$ & $16.2 \pm 1.5$ \\
\hline & $\mathrm{T} 2$ & $252 \pm 56$ & $203 \pm 14$ & $13.7 \pm 1.5$ & $15.9 \pm 1.2$ \\
\hline & $\mathrm{T} 3$ & $259 \pm 65$ & $208 \pm 19$ & $13.7 \pm 1.7$ & $15.7 \pm 1.1$ \\
\hline & $p$ & $\eta_{p}^{2}$ & $p$ & $\eta_{p}^{2}$ \\
\hline \multicolumn{2}{|l|}{ Protocol } & .091 & 0.14 & .117 & 0.11 \\
\hline \multicolumn{2}{|l|}{ Time } & .325 & 0.06 & .163 & 0.09 \\
\hline \multicolumn{2}{|l|}{ Discipline } & .043 & 0.19 & .036 & 0.20 \\
\hline \multicolumn{2}{|c|}{ Protocol $\mathrm{x}$ Time } & .921 & 0.01 & .626 & 0.03 \\
\hline \multicolumn{2}{|c|}{ Protocol $\mathrm{x}$ Discipline } & .034 & 0.21 & .680 & 0.01 \\
\hline \multicolumn{2}{|c|}{ Time $x$ Discipline } & .764 & 0.02 & .444 & 0.04 \\
\hline
\end{tabular}

Values in bold express significant differences between disciplines for the given time point $(p<.05)$. MFBRAKE = braking mean force; T0, pre-effort condition; T1 = postseries 1 condition; $\mathrm{T} 2$ = post-series 2 condition; $\mathrm{T} 3$ = post-series 3 condition.

Power-related variables

An effect of sport discipline was observed on $P_{M I N}$, $M_{\text {TRANSITION, }}$ MP $_{\text {BRAKE }}$ and MPPROP (Table 4). The acyclic group displayed higher positive and lower negative values than the cyclic group in all of these variables. An effect of time was also observed on MPPROP, with the post-hoc test revealing that for the cyclic group MP PROP was significantly lower in T3 than in T0 $(p=.035)$ and T1 $(p=.039)$ during the long protocol.

Velocity-related variables

A similar pattern was exhibited in the variables linked to the velocity component, where the outcomes of the acyclic players were more elevated (higher positive and lower negative) than those of the cyclic athletes (Table 5). An effect of time was observed on $\mathrm{V}_{\mathrm{PP}}\left(F=9.64, p<.001, \eta_{\mathrm{p}}{ }^{2}=0.33\right.$, power $=1.00)$. Post-hoc testing indicated that in the short protocol of the acyclic group $V_{P P}$ was significantly higher during the preeffort condition than during series $2(p=.029)$ and series $3(p$ $=.029$ ), as well as significantly higher in series 1 than in series $3(p=.049)$.

\section{Net impulse}

An effect of the sport discipline was observed on both $\mathrm{NI}_{\text {BRAKE }}$ $\left(F=8.78, p=.008, \eta_{\mathrm{p}}{ }^{2}=0.31\right.$, power $\left.=0.81\right)$ and $\mathrm{NI}_{\mathrm{PROP}}(F=$ $13.20, p=.002, \eta_{\mathrm{p}}^{2}=0.34$, power $\left.=0.86\right)$, with the acyclic group generating a greater net impulse during both sub-phases. In addition, there was a significant effect of time on $\mathrm{NI}_{\text {PROP }}(F$ $=19.07, p<.001, \eta_{p}=0.49$, power $=1.00$ ). Post-hoc testing revealed that in the long protocol of the cyclic group, $\mathrm{NI}_{\mathrm{PROP}}$ was significantly lower during series 3 than in the pre-effort condition $(.=.003$ ) (Table 6$)$.

Rates of force development

RFD UNLOAD, RFD TRANSITION and RFD BRAKE showed significant differences between the cyclic and acyclic groups ( $F=5.13, p$ $=.035, \eta_{p}{ }^{2}=0.20$, power $\left.=0.58\right),\left(F=10.94, p=.004, \eta_{p}{ }^{2}=\right.$ 0.35 , power $=0.88)$ and $\left(F=10.02, p=.005, \eta_{\mathrm{p}}{ }^{2}=0.33\right.$, power $=0.85)$, respectively. Post-hoc tests indicated that the acyclic group displayed higher rates than the cyclic group in all of these measures (Table 6). 
Table 4. Power-related variables. Values are expressed as mean \pm SD

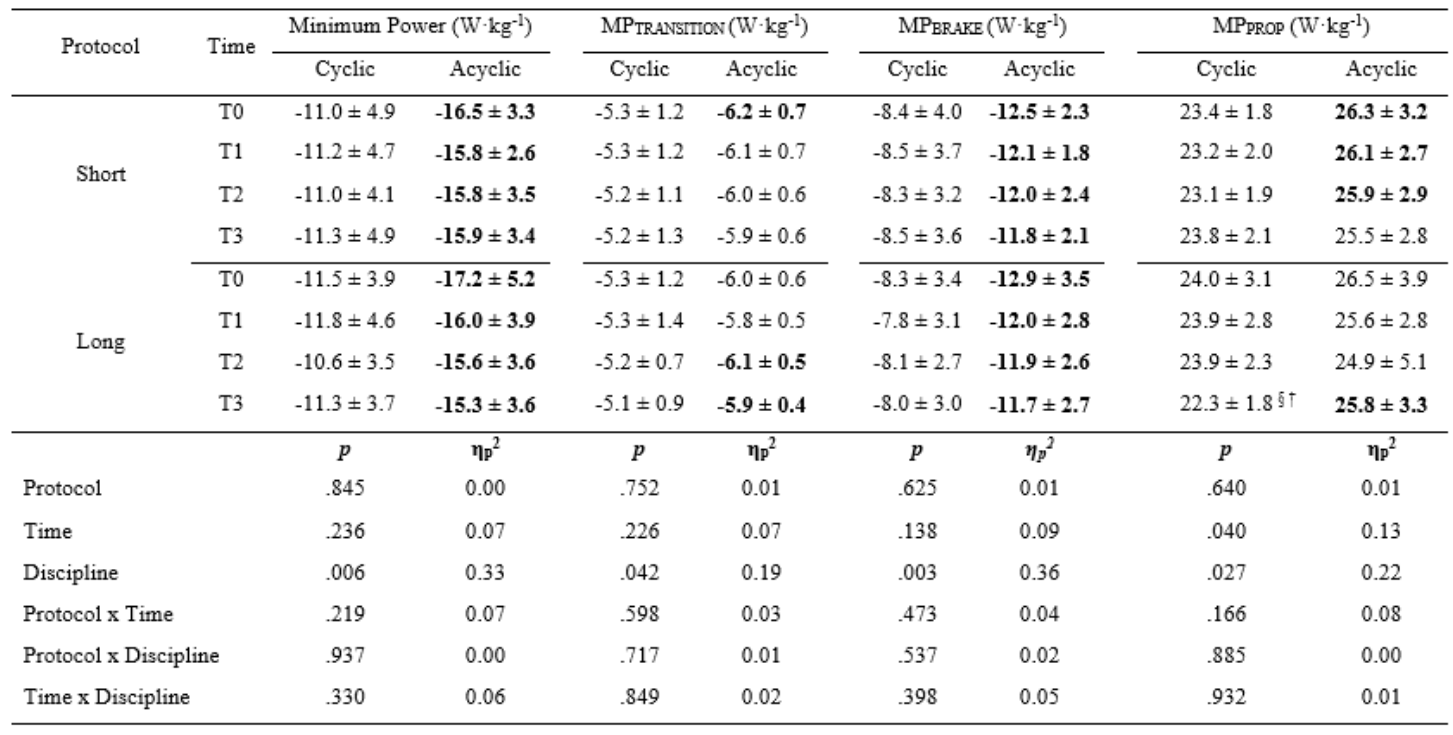

$\S$ Significantly different from T0 $(p<.05)$, $\dagger$ significantly different from T1 $(p<.05)$, Values in bold express significant differences between disciplines for the given time point $(p<.05)$, MPTRANSITION = transition mean power; MPBRAKE = braking mean power; MPPROP = propulsive mean power; $\mathrm{T0}=$ pre-effort condition; $\mathrm{T} 1=$ post-series 1 condition; $\mathrm{T} 2$ = post-series 2 condition; $\mathrm{T} 3$ = post-series 3 condition.

Table 5. Velocity-related variables. Values are expressed as mean \pm SD

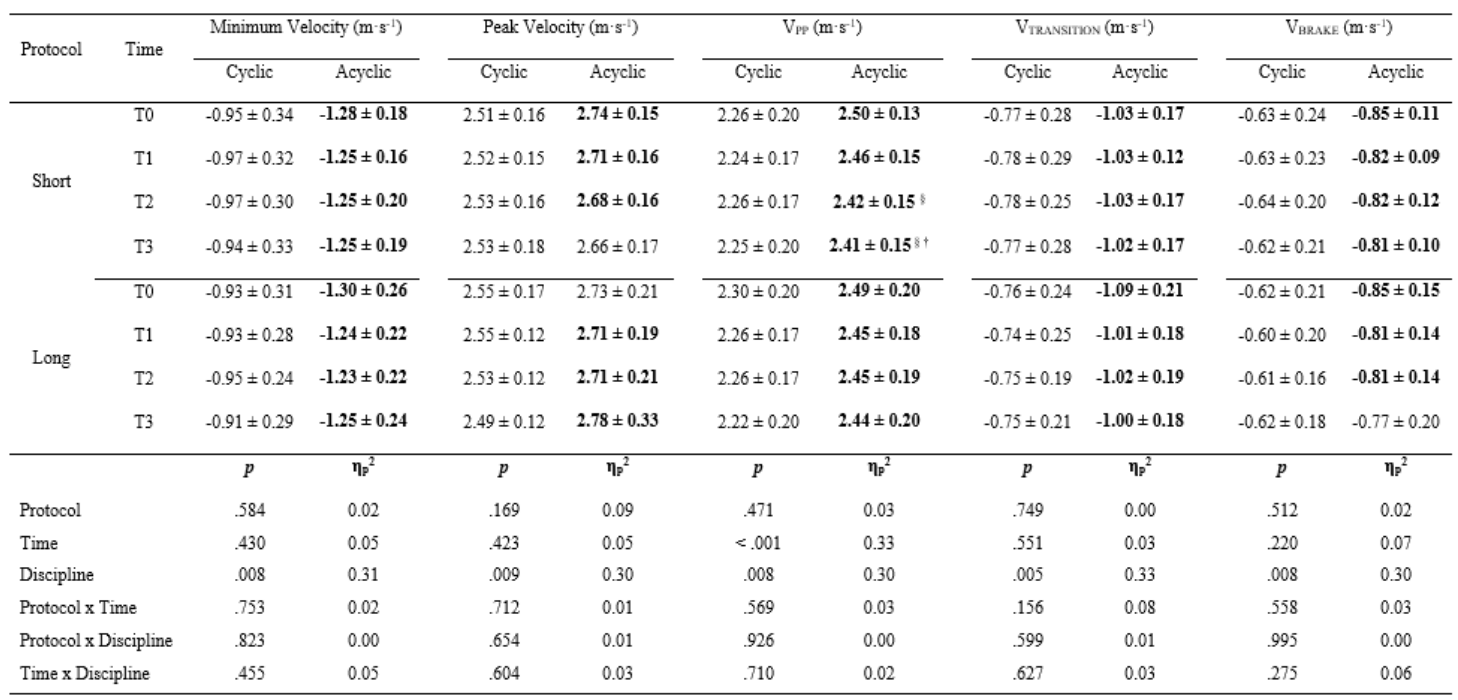

$\S$ Significantly different from T0 $(p<.05)$, † significantly different from T1 $(p<.05)$, Values in bold express significant differences between disciplines for the given time point $(p<.05)$.

$\mathrm{V}_{\mathrm{PP}}=$ velocity at peak power; $\mathrm{V}_{\mathrm{TRANSITION}}=$ transition mean velocity; $\mathrm{V}_{\mathrm{BRAKE}}=$ braking mean velocity; $\mathrm{T} 0$ = pre-effort condition; $\mathrm{T} 1$ = post-series 1 condition; $\mathrm{T} 2$ = postseries 2 condition; $\mathrm{T3}=$ post-series 3 condition.

Cultura, Ciencia y Deporte | AÑo 2022 | volumen $17 \mid$ NUM. 51 | España | PÁG. 131 A 143 | ISSN: 1696-5043 
Analysis of the neuromuscular responses during two different high-intensity interval training sessions in athletes of cyclic and acyclic sports Warr et al.

Table 6. Net impulse and Ratios of force development. Values are expressed as mean \pm SD

\begin{tabular}{|c|c|c|c|c|c|c|c|c|c|c|c|}
\hline \multirow{2}{*}{ Protocol } & \multirow{2}{*}{ Time } & \multicolumn{2}{|c|}{$\mathrm{NI}_{\text {BRAKE }}\left(\mathrm{N} \cdot \mathrm{s} \cdot \mathrm{kg}^{-1}\right)$} & \multicolumn{2}{|c|}{$\mathrm{NI}_{\mathrm{PROP}}\left(\mathrm{N} \cdot \mathrm{s} \cdot \mathrm{kg}^{-1}\right)$} & \multicolumn{2}{|c|}{$\mathrm{RFD}_{\text {UNLOAD }}\left(\mathrm{N} \cdot \mathrm{s}^{-1}\right)$} & \multicolumn{2}{|c|}{$\operatorname{RFD}_{\text {IRAnsmon }}\left(\mathrm{N} \cdot \mathrm{s}^{-1}\right)$} & \multicolumn{2}{|c|}{$\operatorname{RFD}_{\text {BRAKE }}\left(\mathrm{N} \cdot \mathrm{s}^{-1}\right)$} \\
\hline & & Cyclic & Acyclic & Cyclic & Acyclic & Cyclic & Acyclic & Cyclic & Acyclic & Cyclic & Acyclic \\
\hline \multirow{3}{*}{ Short } & T0 & $0.96 \pm 0.34$ & $1.28 \pm 0.18$ & $2.38 \pm 0.20$ & $2.66 \pm 0.17$ & $-1591 \pm 1052$ & $-2465 \pm 907$ & $1954 \pm 947$ & $3266 \pm 982$ & $2739 \pm 1550$ & $4300 \pm 1334$ \\
\hline & $\mathrm{T} 2$ & $0.97 \pm 0.30$ & $1.25 \pm 0.20$ & $2.38 \pm 0.20$ & $2.60 \pm 0.17$ & $-1659 \pm 1057$ & $-2612 \pm 1031$ & $1783 \pm 909$ & $3656 \pm 1521$ & $2204 \pm 1020$ & $4176 \pm 1147$ \\
\hline & T3 & $0.94 \pm 0.34$ & $1.27 \pm 0.22$ & $2.38 \pm 0.20$ & $2.56 \pm 0.22$ & $-1521 \pm 897$ & $-2659 \pm 1104$ & $2017 \pm 1146$ & $3450 \pm 1301$ & $2752 \pm 1661$ & $3798 \pm 1122$ \\
\hline \multirow{3}{*}{ Long } & $\mathrm{T} 1$ & $0.90 \pm 0.30$ & $1.24 \pm 0.22$ & $2.40 \pm 0.15$ & $2.62 \pm 0.20$ & $-1620 \pm 961$ & $-2672 \pm 1032$ & $1899 \pm 1057$ & $3631 \pm 1657$ & $2470 \pm 1737$ & $4239 \pm 1252$ \\
\hline & $\mathrm{T} 2$ & $0.92 \pm 0.24$ & $1.25 \pm 0.23$ & $2.38 \pm 0.15$ & $2.63 \pm 0.23$ & $-1531 \pm 742$ & $-2602 \pm 1058$ & $1860 \pm 835$ & $3454 \pm 1392$ & $2129 \pm 1027$ & $4147 \pm 815$ \\
\hline & T3 & $0.93 \pm 0.27$ & $1.24 \pm 0.22$ & $2.34 \pm 0.148$ & $2.62 \pm 0.23$ & $-1622 \pm 1059$ & $-2693 \pm 1171$ & $1776 \pm 857$ & $3368 \pm 1306$ & $2112 \pm 1275$ & $3982 \pm 695$ \\
\hline & $p$ & $\eta_{\mathrm{p}}^{2}$ & $p$ & $\eta_{\mathrm{p}}^{2}$ & $p$ & $\eta_{\mathrm{p}}^{2}$ & $p$ & $\eta_{\mathrm{p}}^{2}$ & $p$ & $\eta_{\mathrm{p}}^{2}$ \\
\hline \multicolumn{2}{|c|}{ Protocol x Discipline } & .851 & 0.01 & .791 & 0.00 & .740 & 0.01 & .508 & 0.01 & .226 & 0.07 \\
\hline \multicolumn{2}{|c|}{ Time $\mathrm{x}$ Discipline } & .675 & 0.03 & .885 & 0.01 & .790 & 0.02 & .812 & 0.02 & .486 & 0.04 \\
\hline
\end{tabular}

$\S$ Significantly different from TO $(p<.05)$, Values in bold express significant differences between disciplines for the given time point $(p<.05)$, NIBRAKE $=$ braking net impulse; $\mathrm{NI}$ PROP = propulsive net impulse; RFDUNLOAD = unloading rate of force development; RFDTRANSITION $=$ transition rate of force development; RFD $_{\mathrm{BRAKE}}=$ braking rate of force development; $\mathrm{T} 0$ = pre-effort condition; $\mathrm{T} 1$ = post-series 1 condition; $\mathrm{T} 2$ = post-series 2 condition; $\mathrm{T} 3=$ post-series 3 condition.

\section{Discussion}

The purpose of the present study was to determine the effects of short and long HIIT protocols on neuromuscular status assessed through CMJ tests was to analyze the acute effects of a short and a long HIIT session on CMJ performance. As the participants were athletes from different sport modalities, a secondary aim of this study was to compare both the CMJ characteristics and the neuromuscular responses to HIIT between endurance running athletes and team sport players. To our knowledge, this is the first study to analyze the effects of fatigue on jump performance between two HIIT sessions of equal relative load with different work-interval durations. The main findings of this study were a decrease in jump performance due to the total volume completed, irrespective of whether a short or long protocol was conducted, and the fact that specific performance parameters were affected depending on the jumping strategy associated with the sport profile.

Previous studies have addressed the effects of fatigue on neuromuscular function. However, most of them have implemented protocols performed until exhaustion (Bedo et al., 2020; Garrett et al., 2019; Gathercole et al., 2015; Watkins et al., 2017). The present design was based on the completion of regular HIIT prescriptions that are usually conducted in conditioning programs and, consequently, it was not performed until exhaustion. This issue should be considered when interpreting the results of the current study in order to contrast them adequately with those reported in the literature. For example, the $5.7 \%$ reduction in JH observed in the acyclic group at the end of the third series was notably smaller than the $\approx 15 \%$ found in an Australian Football Rules post-match measurement (Garrett et al., 2019) or after a knee extension fatiguing protocol (Rodacki et al., 2002), and smaller than the $10 \%$ reported after 3 consecutive maximal running test (Gathercole et al., 2015). These differences may be explained by the characteristics of the protocols and the total volume applied. In the aforementioned studies, the mean volume reported was $8600 \mathrm{~m}$ (Gathercole et al., 2015) and 12000 m (Garrett et al., 2019) while in the current study the mean volume was almost $5100 \mathrm{~m}$. In addition, the completion of a fatigue protocol focused on specific muscle extenuation (Rodacki et al., 2002), leading to a higher reduction in jump outcomes compared with the present study, where the whole muscular system was involved.

On the other hand, the present findings coincide with those of Thorlund et al. (2008), who reported a 5.2\% reduction in $\mathrm{JH}$ after series of handball-related movements simulating a handball match. This is consistent with the similarities in the configuration of both fatiguing protocols. The total volume of that study was $6500 \mathrm{~m}$, pauses were implemented between repetitions and the total duration was $50 \mathrm{~min}$, parameters that are comparable to the load components of the current study (5100 m, regular pauses and mean total duration of $53.1 \mathrm{~min}$ ).

It has been argued that long HIIT protocols elicit higher lactate and perceptual responses than short-interval prescriptions (Price \& Moss, 2007; Wakefield \& Glaister, 2009; Warr et al., 2018). Therefore, we expected that a significant decrease in jump performance would also be observed in the long protocol than in the short one. Based on the present findings, that hypothesis was rejected, suggesting that CMJ performance during HIIT is not dependent on the metabolic and perceptual parameters.

This paper also explored the jumping strategy applied by groups of sport disciplines with a different performance profile. Previous research has reported a higher $\mathrm{JH}$ for outdoor team sports when compared with indoor team sports, suggesting that the profile of jumpers depended on the sporting background (Laffaye et al., 2014). In the current study, the criterion used to define both groups depended on the sport requirements. Based on this approach, we expected different profiles associated with each group, which were confirmed by the significantly higher speed at maximal oxygen uptake of the cyclic athletes and the significantly higher jumping gross outcomes of the acyclic players.

In addition, differences in CMJ sub-phase characteristics were also found between the cyclic and acyclic groups, revealing that specific jumping patterns were applied by each 
group. The acyclic players executed jumps faster considering the higher values observed in all of the velocity-related variables. This is consistent with previous findings that demonstrated a slower jump contraction associated with groups of lower jump performance outcomes (Jordan et al., 2018; McMahon et al., 2017).

The acyclic group also showed higher values in 4 out of the 5 power-related variables, which is reasonable due to the compound determination of these variables with respect to the velocity component. There were no significant differences in peak power between the cyclic and acyclic groups. This contrasts with the findings of Mc Mahon et al. (2017), who reported a significantly higher peak concentric power in the group with greater jump height (men), attributed to the increased velocity during the concentric phase. An explanation for this discrepancy may lie in both the genderbased criterion used to determine the groups in that study and the smaller amplitude in the peak velocity values between groups observed in the current study.

Relative net impulse plays an important role in determining jumping performance (Kirby et al., 2011). Recent research has shown that a greater impulse during the jump phases was associated with better jumpers (Harry et al., 2018), which is consistent with our findings of significantly higher values in relative net impulse displayed by acyclic players. The rate of force development is another measure that has been shown to be a key determinant of vertical jump performance (Laffaye et al.; 2014, McLellan et al., 2011). However, there were studies that reported no correlation between RFD and vertical jump performance in male soccer players (Barker et al., 2018) and no significant differences between good and poor jumpers (Harry et al., 2018). In the current study, the acyclic group displayed significantly higher RFDs in the three sub-phases for which this measure was calculated, supporting the idea of a possible association between jump performance and RFD in better jumpers. In addition, the fact that differences were observed even in RFD UNLOAD may indicate that a distinct jumping strategy could be applied from the early phases of the jump.

It has been shown that the duration of the CMJ sub-phases alone are weak predictors of jump performance in betweengroup comparisons (Laffaye et al., 2014; Sole et al., 2018) and insufficiently reliable measures in test-retest assessments (Warr et al., 2020). This is consistent with the present findings, which revealed a similar temporal structure exhibited by both groups in all the conditions, except for the duration of the braking sub-phase.

Interestingly, all variables of the braking sub-phase (T $T_{B R A K E}$, $M_{\text {BRAKE, }} V_{\text {BRAKE, }} M_{\text {BRAKE, }} \mathrm{NI}_{\text {BRAKE }}$ and RFD BRAKE) showed significant differences between athletes of cyclic and acyclic sports. This sub-phase reflects the deceleration of the center of mass and provides information on the stretch-shortening cycle (Sole et al., 2018). The explosive characteristics of teamsport movements require an efficient braking capacity prior to the propulsive action. Thus, it should not be surprising that, especially in this sub-phase, acyclic players exhibited higher performance measures than cyclic athletes, since they are familiar with these types of contractions.

Last but not least, the jumping performance was differentially affected by HIIT depending on the sport discipline. Our results suggest that during HIIT, fatigue could start affecting the principal component that the jumping strategy is based on. Acyclic players showed a fast pattern strategy and tended to reduce their velocity when fatigue was accumulated. In contrast, the low braking performance exhibited by the cyclic athletes may indicate that their jumping strategy was based on the propulsive sub-phase, where a decrease in the performance parameters is observed with the reduction of $\mathrm{Nl}_{\text {PROP }}$ and $\mathrm{MP} \mathrm{PROP}_{\mathrm{P}}$ on completion of the long protocol.

There are some aspects of the present study that must be noted as potential limitations. Firstly, as the analyses were based on the VGRF data, no specific information was obtained with regard to the muscular activation amplitude or segmental angular velocities. We have chosen force platform assessments because this is a practical and reliable device that can be easily introduced within the execution of a HIIT session. However, future research should include complementary methods such as electromyography or threedimensional video modelling to determine the effects of HIIT on the neuromuscular function more precisely. The second aspect is that only male regional-level athletes participated in the study. Therefore, the present findings should be interpreted with caution, as it is unclear whether these results would be observed in other male populations (elite athletes, recreational sportsmen) or in females. The last aspect is that the present study focused exclusively on the work-interval duration. The effect that the variation of other components of HIIT could have on neuromuscular performance was beyond the scope of this study. Thus, further research is warranted to better understand the fatigue mechanisms affecting neuromuscular status during different prescriptions of intermittent exercise.

\section{Conclusions}

In summary, this study demonstrated that during one HIIT session, the neuromuscular status was principally affected by the total volume completed rather than by the type of protocol implemented. Specifically, decreases in CMJ performance did not occur until a relatively high volume of HIIT (i.e. three times the $T_{\text {LIM }}$ ) had been completed. In addition, different jumping strategies were applied depending on the profile of the sport discipline. Acyclic players jumped higher than cyclic athletes, this being expressed principally as higher values in the velocity component and the RFD with more efficient performance during the middle sub-phases of the jump (i.e. braking sub-phase). Finally, both groups manifested different responses to fatigue. The acyclic group showed a significant decrease in the jump height outcome, possibly elicited by a reduction of velocity at peak power. In the cyclic group, however, a significant decrease was observed in the net impulse and the mean power of the propulsive sub-phase, even though no reduction in jump height was detected.

\section{Practical Applications}

The present study has shown that HIIT executed at an intensity near the zone of maximal aerobic speed does not affect neuromuscular status until a volume equal to three times the $T_{\text {LIM }}$ is reached. This could be useful for physical trainers and coaches, as it provides specific information about the neuromuscular responses that may be observed when conducting this type of protocols. In several training situations, coaches have to include different physical contents to be conducted within the same session. The current findings indicate that HIIT could be performed concurrently with other skills (i.e. strength training, power training) if a low volume is completed, irrespective of whether a long or short workinterval duration is selected. When the total volume exceeds

Cultura, Ciencia y Deporte $\mid$ AÑo 2022 | volumen 17 | NUM. 51 | España | PÁG. 131 A 143 | ISSN: 1696-5043 
two times the $T_{\text {LIM }}$, then a decrease in jump performance should be expected. In this situation, following the HIIT session, the inclusion of exercises with a high demand in the velocity component are not suggested for acyclic athletes, and exercises with high requirements of propulsive actions (i.e. strength training) should not be implemented in cyclic athletes.

\section{References}

Barker L.A., Harry J.R., \& Mercer J.A. (2018). Relationships between countermovement jump ground reaction forces and jump height, reactive strength index, and jump time. Journal of Strength and Conditioning Research, 32, 248-254. $\mathrm{h}$ ttps://doi.org/10.1519/JSC.0000000000002160

Bedo B.L.S., Pereira D.R., Moraes R., Kalva-Filho C.A., Willde-Lemos T., \& Santiago P.R.P. (2020). The rapid recovery of vertical force propulsion production and postural sway after a specific fatigue protocol in female handball athletes. Gait \& Posture, 77, 52-58. https://doi.org/10.1016/j.gaitpost .2020.01.017

Benítez-Jiménez, A.; Falces-Prieto, M.; García-Ramos, A. (2020). Jump performance after different friendly matches played on consecutive days. Revista Internacional de Medicina y Ciencias de la Actividad Física y el Deporte vol. 20 (77), 185-196 https://doi.org/10.15366/rimcafd2020.77.012

Benjaminse A., Habu A., Sell T.C., Abt J.P., Fu F.H., Myers J.B., \& Lephart S.M. (2008). Fatigue alters lower extremity kinematics during a single-leg stop-jump task. Knee Surgery, Sports Traumatology, Arthroscopy, 16, 400-407. https://doi.o rg/10.1007/s00167-007-0432-7

Billat V.L., Slawinski J., Bocquet V., Demarle A., Lafitte L., Chassaing P., \& Koralsztein J-P. (2000). Intermittent runs at the velocity associated with maximal oxygen uptake enables subjects to remain at maximal oxygen uptake for a longer time than intense but submaximal runs. European Journal of Applied Physiology, 81, 188-196. https://doi.org/1 $0.1007 / \mathrm{s} 004210050029$

Buchheit M. \& Laursen P. (2013). High-intensity training, solutions to the programming puzzle. Part II: anaerobic energy, neuromuscular load and practical applications. Sports Medicine, 43, 927-954. https://doi.org/10.1007/s402 79-013-0066-5

Claudino J.G., Cronin J., Mezêncio B., McMaster D.T., McGuigan M., Tricoli V., Amadio A.C., \& Serrão J.C. (2017). The countermovement jump to monitor neuromuscular status: A meta-analysis. Journal of Science and Medicine in Sport, 20, 397-402. https://doi.org/10.1016/j.jsams.2016.08.011

Cormack S.J., Newton R.U., McGuigan M.R., \& Cormie P. (2008). Neuromuscular and endocrine responses of elite players during an Australian rules football season. International Journal of Sports Physiology and Performance, 3, 439-453. ht tps://doi.org/10.1123/ijspp.3.4.439

Cormie P., McGuigan M.R., \& Newton R.U. (2010). Changes in the eccentric phase contribute to improved stretch-shorten cycle performance after training. Medicine \& Science in Sports \& Exercise, 42, 1731-1744. https://doi.org/10.1249/m ss.0b013e3181d392e8

Dupont G., Blondel N., Lensel G., \& Berthoin S. (2002). Critical velocity and time spent at a high level of for short intermittent runs at supramaximal velocities. Canadian Journal of Applied Physiology, 27, 103-115. https://doi.org/1 0.1139/h02-008
Garrett J., Graham S.R., Eston R.G., Burgess D.J., Garrett L.J., Jakeman J., \& Norton K. (2019). A Novel Method of Assessment for Monitoring Neuromuscular Fatigue in Australian Rules Football Players. International Journal of Sports Physiology and Performance, 14, 598-605. https://doi .org/10.1123/ijspp.2018-0253

Gathercole R., Sporer B., Stellingwerff T., \& Sleivert G. (2015). Alternative Countermovement-jump analysis to quantify acute neuromuscular fatigue. International Journal of Sports Physiology and Performance, 10, 84-92. https://doi.org/10.1 123/ijspp.2013-0413

Griffiths B., Grant J., Langdown L., Gentil P., Fisher J., \& Steele J. (2019). The Effect of In-Season Traditional and Explosive Resistance Training Programs on Strength, Jump Height, and Speed in Recreational Soccer Players. Research Quarterly for Exercise and Sport, 90, 95-102. https://doi.org/ 10.1080/02701367.2018.1563276

Harry J.R., Paquette M.R., Schilling B.K., Barker L.A., James C.R., \& Dufek J.S. (2018). Kinetic and electromyographic subphase characteristics with relation to countermovement vertical jump performance. Journal of Applied Biomechanics, 34, 291-297. https://doi.org/10.1123/ jab.2017-0305

Hori N., Newton R.U., Kawamori N., McGuigan M.R., Kraemer W.J., \& Nosaka K. (2009). Reliability of performance measurements derived from ground reaction force data during countermovement jump and the influence of sampling frequency. Journal of Strength and Conditioning Research, 23, 874-882. https://doi.org/10.1519/JSC.0b013e3 181 a00ca2

Jordan M.J., Aagaard P., \& Herzog W. (2018). A comparison of lower limb stiffness and mechanical muscle function in ACL-reconstructed, elite, and adolescent alpine ski racers/ ski cross athletes. Journal of Sport and Health Science, 7, 416-424. https://doi.org/10.1016/j.jshs.2018.09.006

Kirby T.J., McBride J.M., Haines T.L., \& Dayne A.M. (2011). Relative net vertical impulse determines jumping performance. Journal of Applied Biomechanics, 27, 207-214. https://doi.org/10.1123/jab.27.3.207

Laffaye G., Wagner P.P., \& Tombleson T.I. (2014). Countermovement jump height: Gender and sport-specific differences in the force-time variables. Journal of Strength and Conditioning Research, 28, 1096-1105. https://doi.org/1 0.1519/JSC.0b013e3182a1db03

McLellan C., Lovell D., \& Gass G. (2011). The role of rate of force development on vertical jump performance. Journal of Strength and Conditioning Research, 25, 379-385. https://d oi.org/10.1519/JSC.0b013e3181be305c

McMahon J.J., Rej S.J., \& Comfort P. (2017). Sex differences in countermovement jump phase characteristics. Sports, 5, 8. https://doi.org/10.3390/sports5010008

Moir G.L. (2008). Three Different Methods of Calculating Vertical Jump Height from Force Platform Data in Men and Women. Measurement in Physical Education and Exercise Science, 12, 207-218. https://doi.org/10.1080/10913670802 349766

Price M., \& Moss P. (2007). The effects of work: rest duration on physiological and perceptual responses during intermittent exercise and performance. Journal of Sports Sciences, 25, 1613-1621. https://doi.org/10.1080/02640410 701287248

Rodacki A.L.F., Fowler N.E., \& Bennett S.J. (2002). Vertical jump coordination: fatigue effects. Medicine \& Science in Sports \&

$$
\text { Cultura, Ciencia y Deporte | AÑ̃o } 2022 \text { | vOLUMEN } 17 \mid \text { NUM. } 51 \text { | España | PÁG. } 131 \text { A } 143 \text { | ISSN: 1696-5043 }
$$


Exercise, 34, 105-116. https://doi.org/10.1097/00005768-20 0201000-00017

Rozenek R., Funato K., Kubo J., Hoshikawa M., \& Matsuo A. (2007). Physiological responses to interval training sessions at velocities associated with VO. max. Journal of Strength and Conditioning Research, 21, 188-192. https://doi.org/10.1519 /R-19325.1

Sole C.J., Mizuguchi S., Sato K., Moir G.L., \& Stone M.H. (2018). Phase characteristics of the countermovement jump forcetime curve: A comparison of athletes by jumping ability. Journal of Strength and Conditioning Research, 32, 1155-1165. https://doi.org/10.1519/JSC.0000000000001945

Thorlund J., Michalsik L., Madsen K., \& Aagaard P. (2008). Acute fatigue\#induced changes in muscle mechanical properties and neuromuscular activity in elite handball players following a handball match. Scandinavian Journal of Medicine \& Science in Sports, 18, 462-472. https://doi.org/10 $.1111 /$ j.1600-0838.2007.00710.x

Tschakert G., \& Hofmann P. (2013). High-intensity intermittent exercise: methodological and physiological aspects. International Journal of Sports Physiology and Performance, 8 , 600-610. https://doi.org/10.1123/ijspp.8.6.600

Tschakert G., Kroepfl J., Mueller A., Moser O., Groeschl W., \& Hofmann P. (2015). How to regulate the acute physiological response to "aerobic" high-intensity interval exercise. Journal of Sports Science \& Medicine, 14, 29-36.

Vasquez-Bonilla, A.A., Brazo-Sayavera, J., Timon, R., \& Olcina, G. (2021). Measurement of resting muscle oxygenation and the relationship with countermovement jumps and body composition in soccer players. Cultura, Ciencia y Deporte, 16(48), 215-223. http://dx.doi.org/10.12800/ccd.v16i48.172 6

Wakefield B.R., \& Glaister M. (2009). Influence of WorkInterval Intensity and Duration on Time Spent at a High Percentage VO2max During Intermittent Supramaximal Exercise. Journal of Strength and Conditioning Research, 23, 2548-2554. https://doi.org/10.1519/JSC.0b013e3181bc19b

Warr-di Piero D., Valverde-Esteve T., Redondo-Castán J.C., Pablos-Abella C., \& Sánchez-Alarcos Díaz-Pintado J.V. (2018). Effects of work-interval duration and sport specificity on blood lactate concentration, heart rate and perceptual responses during high intensity interval training. PloS One, 13, e0200690. https://doi.org/10.1371/jo urnal.pone.0200690

Warr D.M., Pablos C., Sánchez-Alarcos J.V., Torres V., Izquierdo J.M., and Redondo J.C. (2020). Reliability of measurements during countermovement jump assessments: Analysis of performance across subphases. Cogent Social Sciences, 6 1843835. https://doi.org/10.1080/23311886.2020.1843835

Watkins C.M., Barillas S.R., Wong M.A., Archer D.C., Dobbs I.J., Lockie R.G., Coburn J.W., Tran T.T., \& Brown L.E. (2017). Determination of vertical jump as a measure of neuromuscular readiness and fatigue. Journal of Strength and Conditioning Research, 31, 3305-3310. https://doi.org/1 $0.1519 /$ JSC.0000000000002231

Wilson J.M., Marin P.J., Rhea M.R., Wilson S.M., Loenneke J.P., \& Anderson J.C. (2012). Concurrent training: a meta-analysis examining interference of aerobic and resistance exercises. Journal of Strength and Conditioning Research, 26, 2293-2307. https://doi.org/10.1519/JSC.0b013e31823a3e2d

Zafeiridis A., Kounoupis A., Dipla K., Kyparos A., Nikolaidis M., Smilios I., \& Vrabas I. (2015). Oxygen delivery and muscle deoxygenation during continuous, long-and short- interval exercise. International Journal of Sports Medicine, 94, 872-880. https://doi.org/10.1055/s-0035-1554634

Cultura, Ciencia y Deporte | AÑo 2022 | volumen 17 | NUM. 51 | España | PÁG. 131 A 143 | ISSN: 1696-5043 